Check for updates

Cite this: RSC Adv., 2019, 9, 14016

Received 23rd February 2019

Accepted 23rd April 2019

DOI: $10.1039 / c 9 r a 01385 g$

rsc.li/rsc-advances

\section{Effect of glyphosate on X-ray diffraction of copper films prepared by electrochemical deposition $\uparrow$}

\author{
Gaomin Zhang, ${ }^{a}$ Bin Xu, ${ }^{\mathrm{b}}$ Hui Chong, ${ }^{\mathrm{a}}$ Wenxian Wei, ${ }^{\mathrm{b}}$ Chengyin Wang (DD *ab \\ and Guoxiu Wang (D)
}

In the process of electrochemical deposition of metals, the additives can directly affect the final morphology of the metal. Using glyphosate as the additive, copper thin films were prepared by the electrochemical deposition method from a $\mathrm{CuSO}_{4}$ aqueous solution under a specific voltage. The copper thin films were grown on the surface of the indium tin oxide (ITO) film, which was used as the working electrode in a classical threeelectrode cell. Glyphosate combined with the copper ion to form a complex, and hindered further reduction and crystallization of the copper ions. The results indicated that the peak intensities of the $\mathrm{X}$-ray diffraction peaks decreased with the increase in the glyphosate concentrations, which can be used as a basis for quantitative detection. The method is simple and highly sensitive.

\section{Introduction}

Glyphosate is regarded as one of the most effective nonelectroactive organophosphorus herbicides in agricultural production and landscape maintenance because of its ability to control weeds effectively. ${ }^{1,2}$ However, the unlimited usage of the glyphosate has detrimental impacts on animals, aquatic plants, microbial compositions and potential indirect effects on human health., Therefore, a sensitive detection of glyphosate is highly demanded. Until now, the reliable detection methods for glyphosate are the chromatographic methods including gas chromatography tandem mass spectrometry (GC-MS), ${ }^{\mathbf{5} \mathbf{6}}$ liquid chromatography tandem mass spectrometry (LC-MS) ${ }^{7-9}$ and chemical sensing methods. ${ }^{10-12}$ Despite high sensitivity and promising stability, the chromatographic methods require complicated sample pre-treatment and chemical derivatization processes to facilitate the detection or the separation of the analytes. ${ }^{13}$ Meanwhile, the complicated fabrication process of the highly sensitive glyphosate chemical sensor hindered its wide applications. Currently, a simple and convenient operation is regarded as the design principle for the glyphosate sensing. Thus, the development of a novel detection method for glyphosate with a highly sensitive and facile operation remains challenging. To overcome the disadvantages of the traditional chromatography and the chemical sensing methods, we attempted to establish a highly sensitive and facile glyphosate detection approach via alternative technologies.

${ }^{a}$ College of Chemistry and Chemical Engineering, Yangzhou University, Yangzhou 225002, China.E-mail: wangcy@yzu.edu.cn

${ }^{b}$ Testing Center, Yangzhou University, Yangzhou 225009, China

${ }^{c}$ Centre for Clean Energy Technology, Faculty of Science, University of Technology Sydney, Sydney, NSW, Australia. E-mail: Guoxiu.Wang@uts.edu.au

$\dagger$ Electronic supplementary information (ESI) available. See DOI: 10.1039/c9ra01385g
Electrochemical deposition is one of the most extensively used approaches to prepare metals, metal oxides and metal based functional materials, ${ }^{\mathbf{1 4}}$ including coatings in the electronics industry, functional nanomaterials and the multicomposite electrode materials. ${ }^{15-20}$ The electrochemical deposition has the advantages of utilizing simple equipments and operation, mild conditions, high controllability, requiring no separation and high stability. The morphology control can be achieved by the application of the additives during the deposition process. ${ }^{21-23}$ For instance, branched polyethyleneimine (BPEI) was reported to affect the morphology and the electrodeposition kinetics of zinc in $\mathrm{ZnSO}_{4}$ solution. The presence of BPEI could alter the morphology of the electrodeposited layer from laminated hexagonal large crystals to an amorphous compact layer. ${ }^{24}$ Organic additives including cresol, resorcinol and synthetic alcohol affected the deposition process of $\mathrm{Bi}^{3+}$ ions in acidic perchlorate solution, and produced dense and uniform coatings. ${ }^{25}$ The influence of the electrolyte additives on the shape and the morphology of nanocrystals in the electrodeposited copper films has been investigated. ${ }^{26}$ These organic additives were adsorbed on the active sites on the surface of the electrode and combined with the metal ions to form complexes that changed the nucleation and growth of the crystals. ${ }^{27-29}$ The microstructure and the morphology of the deposited metal change, and the X-ray diffraction pattern changes accordingly. Differences in the concentration or the type of the additives resulted in the different changes in the X-ray diffraction pattern. $\mathrm{X}$-ray diffraction measurement is one of the necessary steps in the characterization of these electrodeposited materials due to the successful elucidation of the crystal structures from the diffraction patterns. ${ }^{30}$ The technique can provide complete crystalline structural information including cell parameters, grain size, microscopic stress, lattice constant, and preferred 
crystal orientation. ${ }^{31,32}$ It shows the advantages of negligible sample damage, non-pollution, short detection time and high accuracy. ${ }^{33}$

Inspired by this additive resulted crystal and morphology change phenomenon, we attempted to establish a novel glyphosate detection method based on the combination of the electrochemical deposition and the X-ray diffraction technologies. The negatively charged phosphate group from glyphosate allowed the formation of the complex with the positively charged metal ions. This reaction provided a basis for the crystal and the morphological changes of the metal electrodeposited films. We chose copper as the depositing metal as it was reported that glyphosate had a strong interaction with $\mathrm{Cu}^{2+}$ ions. ${ }^{34}$ Compared to the precious metals like platinum, gold and their alloys, the transition metal copper is inexpensive and cannot be easily oxidized. Copper can be electrochemically deposited directly at a constant potential. The different concentrations of glyphosate were added as additives into the $\mathrm{CuSO}_{4}$ electrolyte solution. Due to the change in the electrolyte composition, $\mathrm{Cu}^{2+}$ combined with glyphosate. This process led to the change in the microstructure and the deposition morphology of the copper films. Accordingly, the intensity of diffraction peaks exhibited significant changes. In summary, this method extended the range of the traditional quantitative $\mathrm{X}$-ray diffraction measurements and has a wide application prospect in the detection of the non-electroactive pesticide glyphosate.

\section{Experiment section}

\subsection{Reagents and materials}

Glyphosate ( $N$-(phosphonomethyl)glycine), copper sulfate pentahydrate, and sulfuric acid were purchased from Sinopharm Chemical Reagent Co. Ltd. Indium tin oxide (ITO) glass slides were purchased from Zhuhai Kaivo Electronic Components Co. The platinum electrode and a saturated calomel electrode were purchased from Nanjing Cole equipment Co. Ltd. All reagents were of analytical reagent grade. The water used was purified through a Millipore system.

\subsection{Electrochemical deposition}

In this experiment, a classical three-electrode system composed of the conductive Indium tin oxide (ITO), a platinum electrode and a saturated calomel electrode (SCE) was adopted for the electrochemical deposition reaction. Before deposition, the ITO substrates were cleaned in acetone, ethanol and deionized (DI) water for $5 \mathrm{~min}$ each in an ultrasonic bath. The working electrode (WE) was the cleaned ITO substrate with a working area of $1.0 \mathrm{~cm} \times 3.0 \mathrm{~cm}$. The conductive layer on the surface of the ITO substrate was indium tin oxide. The thickness of the ITO and its film were $1.1 \mathrm{~mm}$ and $185 \mathrm{~nm}$, respectively. The saturated calomel electrode (SCE) was used as the reference electrode (RE) and a platinum electrode worked as the counter electrode (CE). The electrolyte solutions were prepared from $0.010 \mathrm{~mol} \mathrm{~L}^{-1}$ $\mathrm{CuSO}_{4}$ and $0.10 \mathrm{~mol} \mathrm{~L}^{-1} \mathrm{H}_{2} \mathrm{SO}_{4}$ solutions. Copper films were deposited on the ITO substrate surface from the electrolyte with the temperature maintained at $25{ }^{\circ} \mathrm{C}$. In the subsequent experiments, the copper concentration was kept at $0.010 \mathrm{~mol} \mathrm{~L}^{-1}$. The glyphosate solution was added to the $\mathrm{CuSO}_{4}$ aqueous solution as the additive in different concentrations between $1.0 \times 10^{-5} \mathrm{~mol} \mathrm{~L}{ }^{-1}$ and $1.0 \times 10^{-10} \mathrm{~mol} \mathrm{~L}^{-1}$. The electrodeposition was performed at a constant applied potential $(-0.80 \mathrm{~V})$ and the deposition time was 20 minutes. The $\mathrm{pH}$ of the electrolyte was kept at 3.50. After the copper films fully covered the ITO substrate surface, the samples were rinsed with deionized water to remove residual sulfates from the surface of the copper films, and then dried at room temperature.

\subsection{Apparatus}

The electrochemical deposition was carried out on an electrochemical workstation (CHI 660) (Shanghai Chenhua Instrument Co., China). X-ray diffraction was performed on a D8 Advance polycrystalline X-ray diffractometer (Bruker-AXS, Germany) using $\mathrm{Cu} \mathrm{K}_{\alpha}$ radiation. The X-ray diffraction spectra obtained in the experiment were all separated by $\mathrm{K}_{\alpha 1}$ and $\mathrm{K}_{\alpha 2}$. The morphologies of the copper films were characterized by scanning electron microscopy (SEM, Hitachi S-4800, Japan) at an accelerating voltage of $30 \mathrm{kV}$. Energy dispersive X-ray spectroscopy (EDX) analyses were carried out with an EDAX instrument (Genesis XM2, USA). Transmission electron microscopy (TEM) was performed on an FEI Tecnai F30 TEM transmission electron microscope operating at an accelerating voltage of $300 \mathrm{kV}$. The valences of copper ion were calculated by X-ray photoelectron spectroscopy (XPS, VG 2000) using Al $\mathrm{K}_{\alpha}$ monochromated radiation as the exciting source.

\section{Results and discussions}

\subsection{Structural and morphological characterizations of copper films}

The surface SEM studies were conducted to understand the difference in the surface morphologies and the effect of glyphosate on the deposited copper films. The results are displayed in Fig. 1. It can be seen from the SEM images that the deposited pure copper films (Fig. 1a) could form typical three-dimensional dendritic structures on the ITO surface. With the addition of glyphosate in the electrolyte (Fig. 1b), the dendritic structures increased significantly compared with that of pure copper films, indicating a small crystallite size. ${ }^{35}$ This showed that the addition of glyphosate could refine the deposited copper films, change the morphologies of the deposited coatings, and affect the growth of the copper crystals. From the TEM images, it was more evident that the crystallite size of the deposited copper films with the existence of glyphosate (Fig. 2b) was smaller than that of the pure copper films (Fig. 2a).

In order to provide detailed data for the influence on the copper crystallite growth in the presence of glyphosate, we conducted the X-ray diffraction measurements of the copper film synthesized with and without the addition of glyphosate. As shown in Fig. 3a, without the addition of glyphosate, the copper film showed three $(h k l)$ peaks with $2 \theta$ values of $43.34^{\circ}, 50.23^{\circ}$, and $73.90^{\circ}$, which could be assigned to the (111), (220) and (200) 


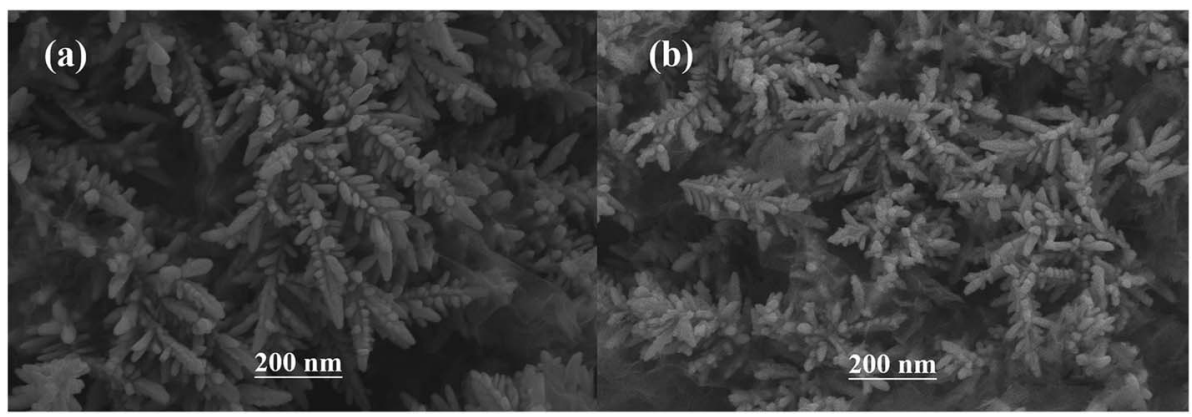

Fig. 1 SEM images of the copper films on the ITO substrate surface electrodeposited without (a) and with glyphosate additives (b). The concentration of glyphosate was $1.0 \times 10^{-5} \mathrm{~mol} \mathrm{~L}-1$, and the copper concentration was $0.010 \mathrm{~mol} \mathrm{~L}^{-1}$.

facets of copper, respectively. Among them, the peak with the $2 \theta$ value of $43.34^{\circ}$ showed the strongest intensity and it was consistent with the standard diffraction card (PDF 65-7743), indicating that the deposited copper films have the face centered cubic structure. We also concluded from the X-ray diffraction pattern that the electro-crystallization of the copper was in a lateral growth manner, which indicated a tightly packed atomic plane with the $c$-axis oriented parallel to the substrate surface. The rest of the peaks with low intensity observed in the X-ray diffraction pattern could be assigned to indium tin oxide $\left(\mathrm{In}_{4} \mathrm{Sn}_{3} \mathrm{O}_{12}\right)$ and silicon dioxide $\left(\mathrm{SiO}_{2}\right)$ from the supporting ITO glass slide. As shown in Fig. 3b, upon the addition of glyphosate during the process of electrochemical deposition, the intensities of the (111), (220) and (200) peaks decreased in contrast to the peak intensities of pure copper films electro-deposited without glyphosate. This intensity reduction was also seen when the intensity of ITO glass was used as the reference. The reason for the peak intensity reduction was proposed to be the addition of the glyphosate significantly affecting the electrochemical deposition of the copper, which was probably due to the complexation of $\mathrm{Cu}^{2+}$ with glyphosate in the electrolyte, resulting in a decrease in the copper electro-crystallization during the normal electrochemical deposition.
Subsequently, X-ray diffraction measurements of copper films with the addition of varied concentrations of glyphosate were conducted to investigate the influence of the additive concentrations on the copper crystallite growth. The effect of different concentrations of glyphosate on X-ray diffraction patterns is presented in Fig. 4 . It could be observed that the increase in the concentration of glyphosate in the electrolyte reduced the (111)-preferred orientations and the intensities of the diffraction peaks. Therefore, it was reasonable to deduce that the concentration of glyphosate was closely related to the preferred orientation of the deposited copper films and the intensities of X-ray diffraction peaks. The texture coefficient (TC) of each lattice plane was estimated to characterize the preferred orientation using the equation presented below:

$$
\mathrm{TC}_{h k l}=\frac{I_{(h k l)} / I_{0(h k l)}}{\sum_{i=1}^{n} I_{(h k l)} / I_{0(h k l)}} \times 100 \%
$$

where $I_{(h k l)}$ was the intensity of the diffraction peak measured in the deposited sample, $I_{0(h k l)}$ was the diffraction peak intensity of a standard $\mathrm{Cu}$ powder. $\mathrm{TC}_{h k l}$ was the texture coefficient and $n$ was the number of diffraction peaks. The larger the TC value of a lattice plane, the higher the preferred orientation of the lattice plane and the greater the intensity of the diffraction peak. The

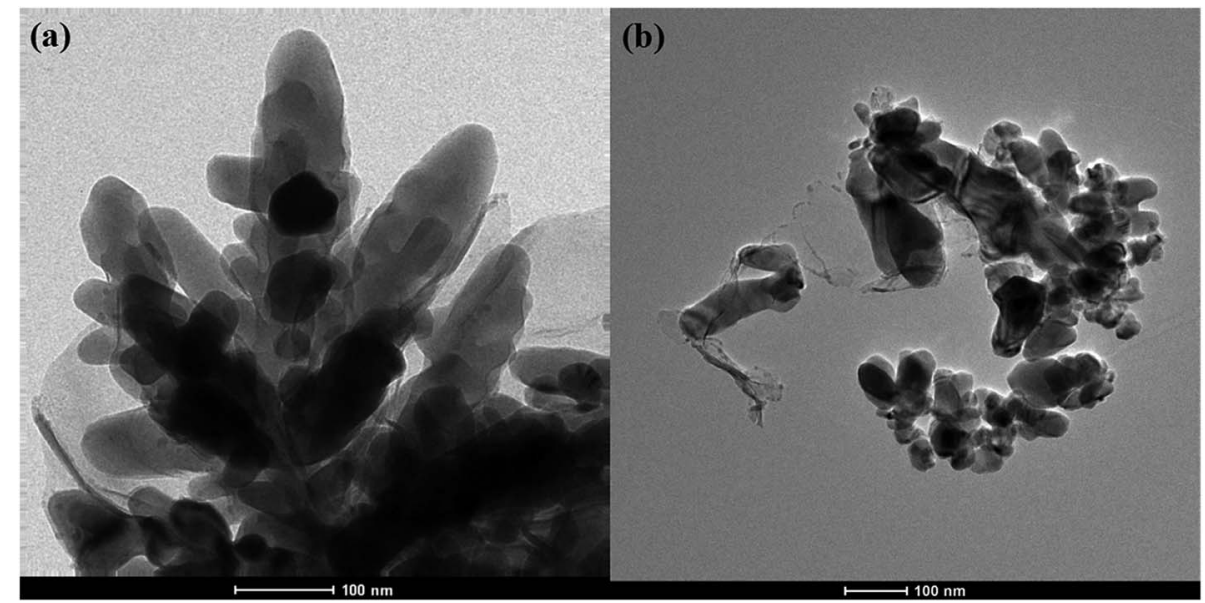

Fig. 2 TEM images of the copper films on the ITO substrate surface electrodeposited without (a) and with glyphosate additives (b). The concentration of glyphosate was $1.0 \times 10^{-5} \mathrm{~mol} \mathrm{~L}^{-1}$, and the copper concentration was $0.010 \mathrm{~mol} \mathrm{~L}^{-1}$. 

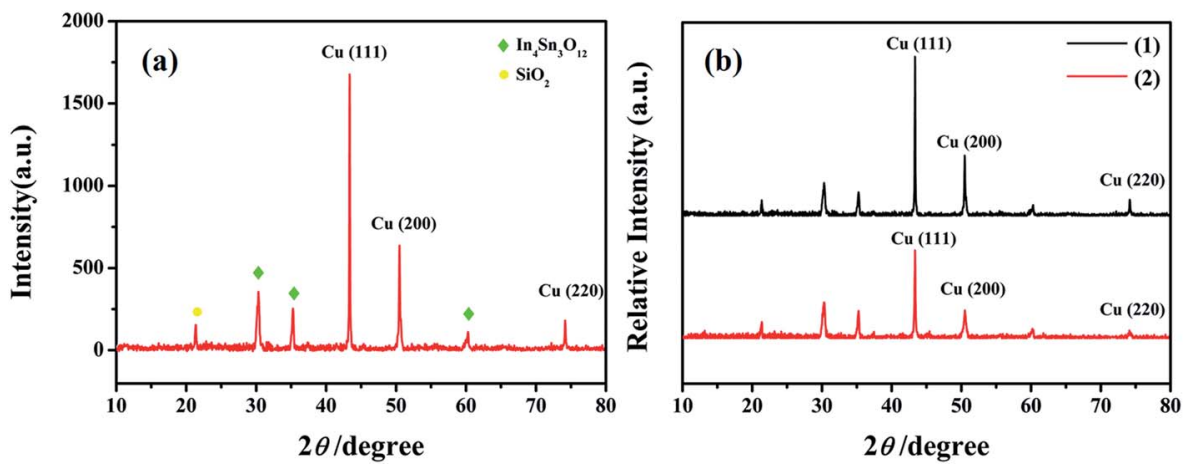

Fig. 3 X-ray diffraction patterns of the pure copper films (a) and the changes of the relative intensities of the copper films (b) electrodeposited without (1) and with the glyphosate additives (2).

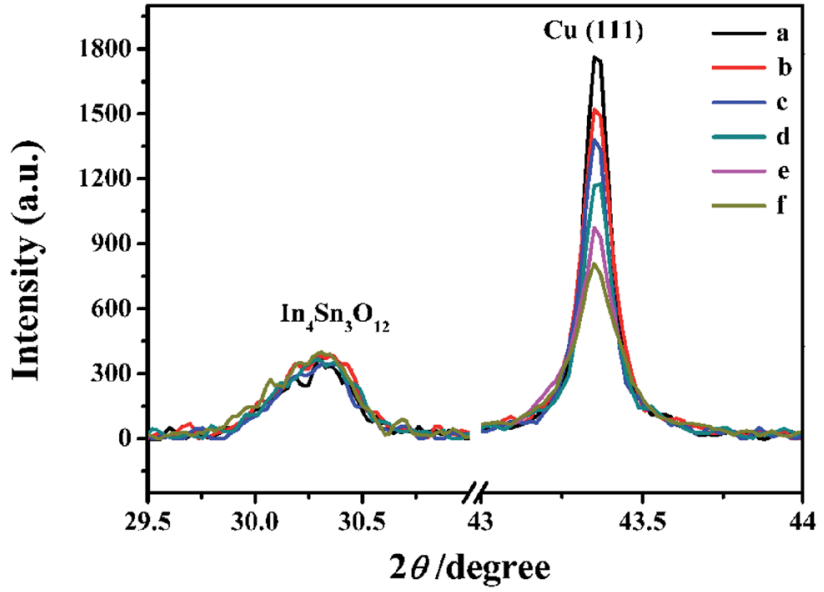

Fig. 4 X-ray diffraction patterns of the electrodeposited copper films with the addition of glyphosate in different concentrations. The concentrations of the glyphosate were (a) $1.0 \times 10^{-10} \mathrm{~mol} \mathrm{~L}^{-1}$, (b) 1.0 $\times 10^{-9} \mathrm{~mol} \mathrm{~L}^{-1}$, (c) $1.0 \times 10^{-8} \mathrm{~mol} \mathrm{~L}^{-1}$, (d) $1.0 \times 10^{-7} \mathrm{~mol} \mathrm{~L}^{-1}$, (e) $1.0 \times$ $10^{-6} \mathrm{~mol} \mathrm{~L}^{-1}$, (f) $1.0 \times 10^{-5} \mathrm{~mol} \mathrm{~L}^{-1}$. The copper concentration was kept at $0.010 \mathrm{~mol} \mathrm{~L}^{-1}$.

results of the TC values are presented in Table 1 . It can be seen from the Table 1 that all the electrodeposited copper films showed the high (111)-preferred orientation under the influence of different concentrations of glyphosate. Therefore, the effect of glyphosate on the intensities of the electrodeposited copper films was the most pronounced based on the high (111)preferred orientations. In this work, the effect of glyphosate on the intensities of X-ray diffraction peaks was studied on the basis of the changes of the (111) lattice plane intensities.

Fig. 4 shows the changes in peak intensities of (111) lattice planes in the X-ray diffraction pattern of the deposited copper films after adding different concentrations of glyphosate. The addition of the glyphosate with evaluated concentrations resulted in the gradual reduction of the copper diffraction peak intensity using ITO diffraction intensity as the reference. Compared with other samples, the diffraction peak intensity of the deposited copper films without glyphosate was the maximum and the peak intensities of the (111) lattice planes gradually decreased with the increase in the glyphosate concentrations. Hence, it was plausible to infer that the addition of glyphosate significantly affected the electrochemical deposition of copper, which was probably due to the complexation of $\mathrm{Cu}^{2+}$ with glyphosate in the electrolyte, resulting in a decrease in the copper electro-crystallization during normal electrochemical deposition. Distinctly, the variations in the $\mathrm{X}$ ray diffraction peak intensities could not only reflect the presence of glyphosate, but also be utilized for the quantitative detection of glyphosate in the sample.

Rietveld refinement was employed by TOPAS software for the unit cell parameter analysis of the deposited copper films. In the Rietveld refinement process, $R_{\mathrm{wp}}$ indicated the radiation work permit and was used to evaluate the fitting results. Generally, when $R_{\mathrm{wp}}$ was less than 15 , the result could be considered as reliable. It can be seen from the results in Table 2 that the calculated $R_{\mathrm{wp}}$ values were all less than 15 . The

Table 1 Effect of different concentrations of glyphosate on the TC value of deposited copper films $(n=3)^{a}$

\begin{tabular}{|c|c|c|c|c|}
\hline 1 & 0 & $44.30 \pm 0.52$ & $37.32 \pm 0.63$ & $18.38 \pm 0.11$ \\
\hline 3 & $1.0 \times 10^{-9}$ & $47.63 \pm 0.30$ & $33.89 \pm 0.46$ & $18.48 \pm 0.16$ \\
\hline 4 & $1.0 \times 10^{-8}$ & $49.01 \pm 0.47$ & $34.75 \pm 0.56$ & $16.24 \pm 0.19$ \\
\hline 5 & $1.0 \times 10^{-7}$ & $47.68 \pm 0.53$ & $36.09 \pm 0.65$ & $16.23 \pm 0.13$ \\
\hline
\end{tabular}

${ }^{a} n$ is the repetitive measurements number. 
Table $2 R_{\mathrm{wp}}$ values of the deposited copper samples containing different concentrations of the glyphosate $(n=3)^{a}$

\begin{tabular}{lll}
\hline Number of samples & $\begin{array}{l}\text { Concentrations of } \\
\text { glyphosate }(\mathrm{M})\end{array}$ & $R_{\mathrm{wp}}$ \\
\hline 1 & 0 & $6.94 \pm 0.40$ \\
2 & $1.0 \times 10^{-10}$ & $5.75 \pm 0.25$ \\
3 & $1.0 \times 10^{-9}$ & $6.25 \pm 0.67$ \\
4 & $1.0 \times 10^{-8}$ & $7.03 \pm 0.94$ \\
5 & $1.0 \times 10^{-7}$ & $9.16 \pm 0.95$ \\
6 & $1.0 \times 10^{-6}$ & $6.86 \pm 0.99$ \\
7 & $1.0 \times 10^{-5}$ & $7.49 \pm 0.73$ \\
${ }^{a} n$ is the repetitive measurements number.
\end{tabular}

calculated $R_{\mathrm{wp}}$ results indicated that the experimental raw data and the fitting data had less fitting error.

The effect of different concentrations of glyphosate on the unit cell parameters has been presented in Fig. 5. According to the unit cell parameters presented in Fig. 5, the result of unit cell parameters indicated that the crystallite size, cell volume and the lattice constant $(a)$ decreased with the increasing concentration of the glyphosate in deposited copper films. A small increase in these unit cell parameters could be possible due to the glyphosate incorporation in the copper structure/ film. This outcome was in agreement with the previously obtained results of the SEM and TEM images.

\subsection{Chemical characterization}

Energy Dispersive X-ray Spectrometry EDX-Mapping technique was used to analyze the distribution characteristics of the elements in the electrodeposited copper films in the presence of glyphosate. The copper films containing glyphosate was scraped from the surface of the ITO and dispersed ultrasonically in an ethanol solution. The crystal containing dendritic structure and amorphous structure was taken from the electrodeposited copper films for EDX energy spectrum analysis. Fig. 6a shows the TEM topography, which could be divided into two groups according to the structural features in the figure. The dendritic structure indicates area 1 and the amorphous structure
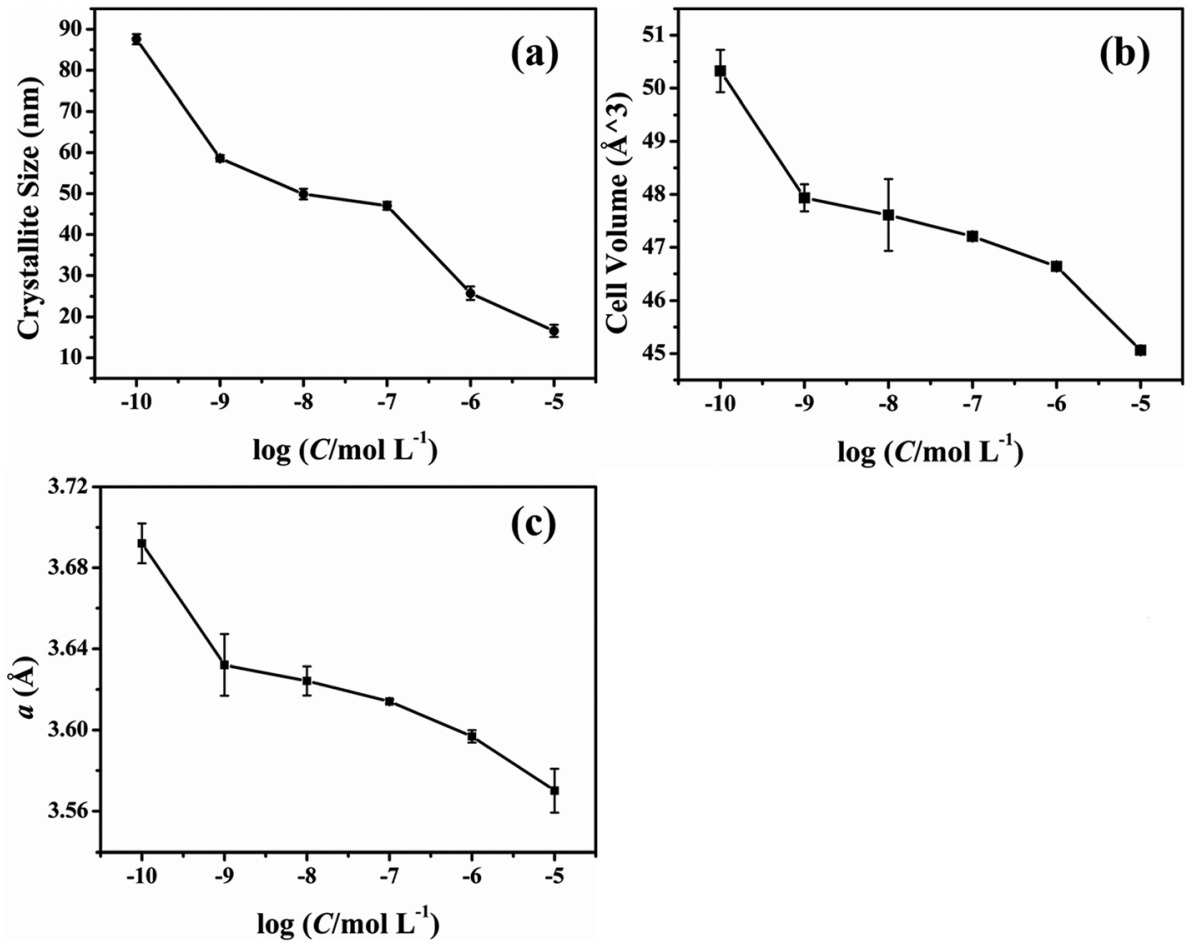

Fig. 5 Effect of the glyphosate concentration on the unit cell parameters of the deposited copper films.
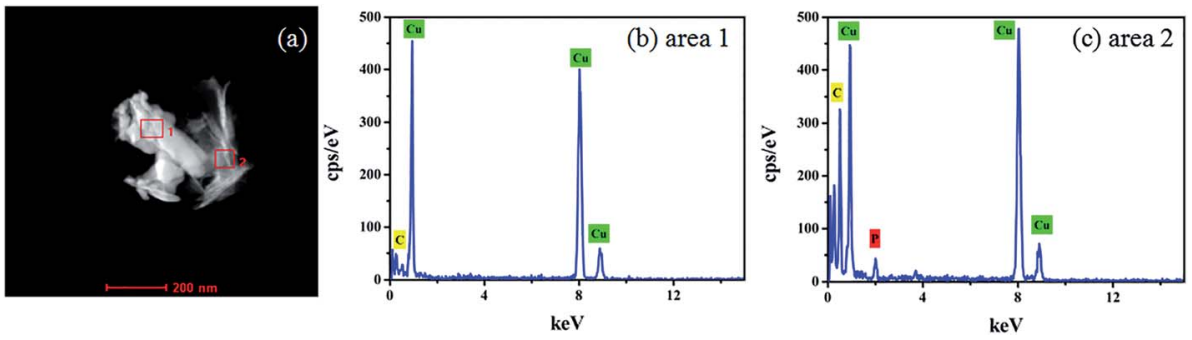

Fig. 6 EDX spectrum of the electrodeposited copper films with glyphosate additives for area 1 and area 2. 
indicates area 2. According to the recorded peak values, the copper peaks could be distinguished from the EDX spectrum of area 1 (Fig. 6b). Moreover, apart from copper peaks, the peak of phosphorus was also recognized in the spectrum of area 2 (Fig. 6c). The results directly provided evidence that phosphorus was incorporated into the crystal structure of copper. Specifically, the $\mathrm{Cu}^{2+}$ ions in the electrolyte were reduced to pure copper metal at the cathode, while a fraction of $\mathrm{Cu}^{2+}$ ions complexed with the phosphorus ions, after which the products were deposited on the ITO substrate surface.

According to the results displayed in Fig. 7, the yellow part of Fig. $7 \mathrm{~b}$ is the distribution of $\mathrm{Cu}$ elements in the sample, and the red part of Fig. 7c shows the distribution of phosphorus elements. The result demonstrated that the phosphorus mainly accumulated on the amorphous structure of the electrocrystallized copper rather than the dendritic structure. Combined with the EDX spectrum, it could be inferred that the complex product of $\mathrm{Cu}^{2+}$ ions and phosphorus was more likely to form an amorphous structure than the dendritic structure on the substrate surface.

In order to investigate the mechanism of the effect of glyphosate on the intensities of X-ray diffraction peaks, the valence states of copper after the addition of glyphosate was studied by X-ray photoelectron spectroscopy (XPS). In this experiment, the peak of carbon atom at $231 \mathrm{eV}$ was used as the reference to calibrate the binding energy (BE). During the measurement, the adventitious carbon could not be completely removed, and the residual amounts affected the results of our

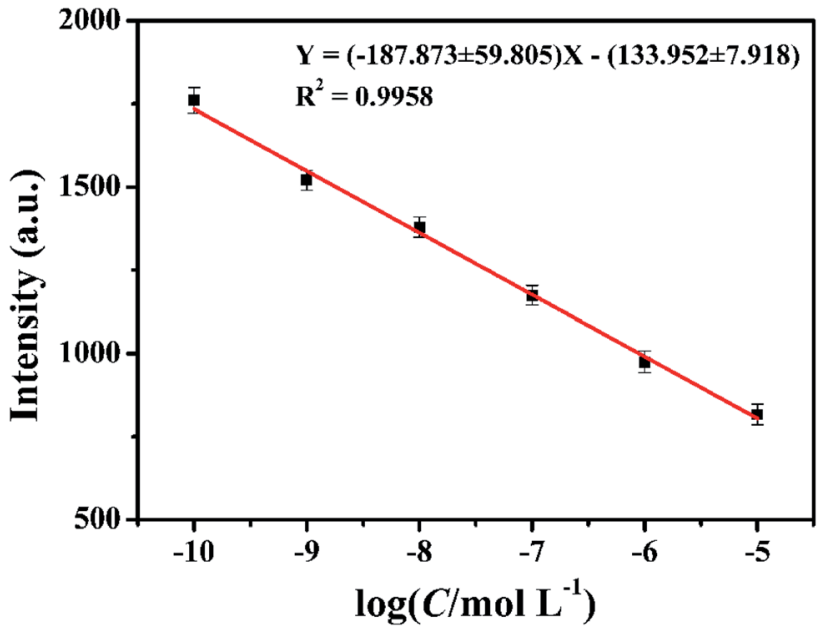

Fig. 9 The corresponding calibration curve for the glyphosate determination. The copper concentration was $0.01 \mathrm{~mol} \mathrm{~L}^{-1}$.

XPS measurements. As shown in Fig. 8a, P, C, N, O and $\mathrm{Cu}$ elements were detected in the XPS spectra of the electrodeposited copper film formed in the presence of glyphosate. Fig. $8 \mathrm{~b}$ shows the XPS spectrum of the $\mathrm{Cu}-2 \mathrm{p}$ core region. After the complexation of $\mathrm{Cu}^{2+}$ ion with glyphosate, the XPS spectrum of the $\mathrm{Cu}-2 \mathrm{p}$ region split into two absorption peaks at $935.0 \mathrm{eV}$ and $932.3 \mathrm{eV}$. The peak at $935.0 \mathrm{eV}$ corresponded to the $\mathrm{Cu}-2 \mathrm{p}_{3 / 2}$ core level of $\mathrm{Cu}^{2+}$ ions, which was physically adsorbed on the ITO substrate surface. The binding energy of $932.3 \mathrm{eV}$
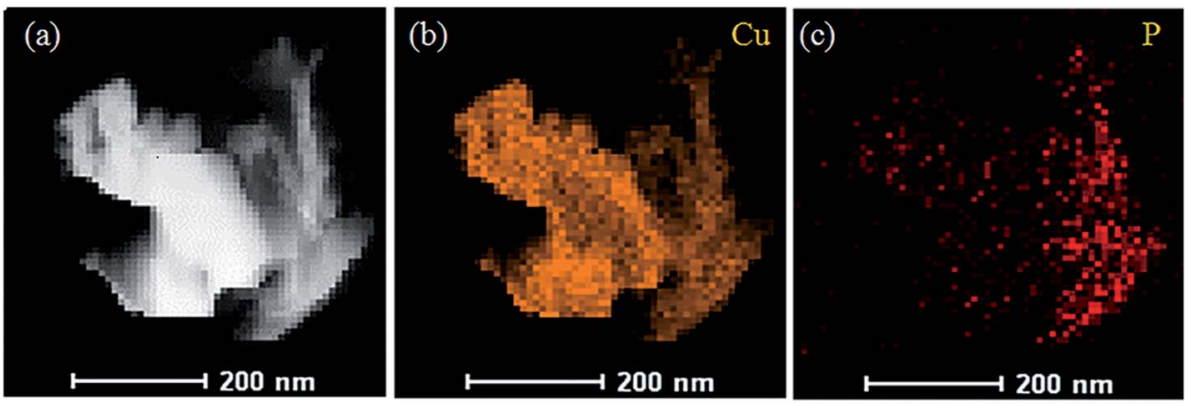

Fig. 7 Mapping images of the elemental distributions of the deposited copper films added with glyphosate.
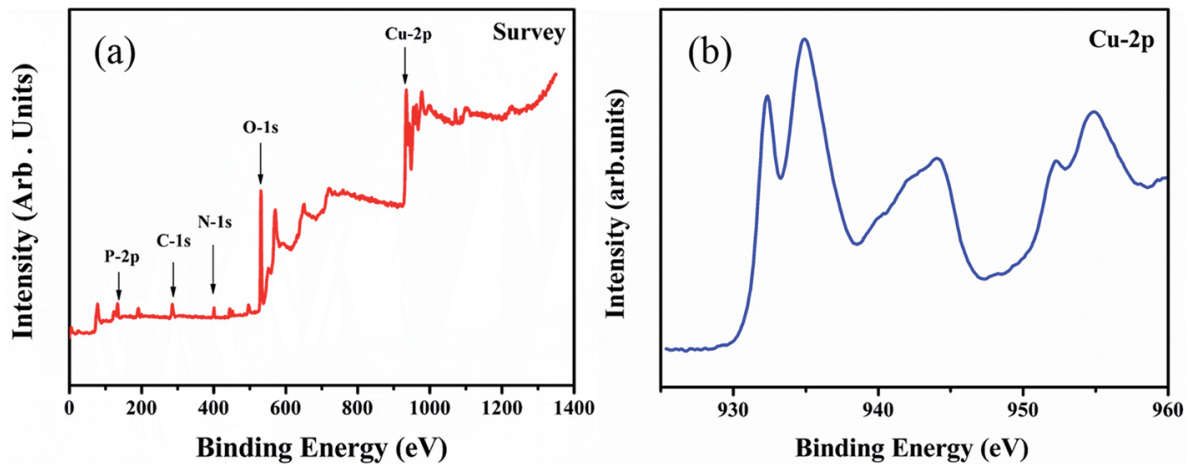

Fig. 8 XPS spectra corresponding to the (a) deposited copper films with the addition of glyphosate, (b) Cu-2p core level regions of copper films supported on the ITO substrate. 
Table 3 Recovery measurements of glyphosate in milk $(n=3)^{a}$

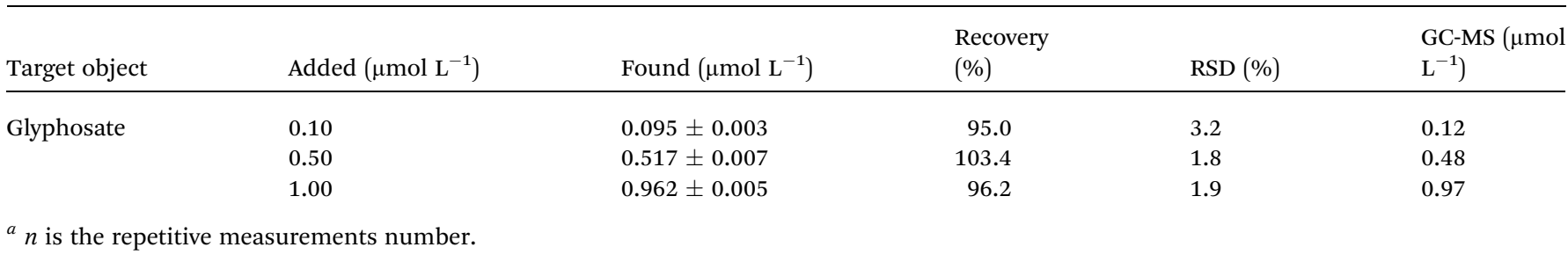

represented the $\mathrm{Cu}^{2+}$ ions that coordinated with glyphosate adsorbed on the surface of ITO because $\mathrm{P}=\mathrm{O}$ bond in the glyphosate structure had strong affinity towards the $\mathrm{Cu}^{2+}$ ions. ${ }^{34}$ The $\mathrm{d}^{9}$ electronic configuration of $\mathrm{Cu}^{2+}$, in which one electron could be easily excited to the $4 \mathrm{p}$ orbital to form a dsp ${ }^{2}$ hybrid orbital, facilitated the formation of the complex between ligands by accepting lone pair of electrons. The reduction of binding energy from $935.0 \mathrm{eV}$ to $932.3 \mathrm{eV}$ indicated that there was an electron density change for $\mathrm{Cu}^{2+}$ ions during complexation. The complex formed subsequently deposited on the surface of the ITO and hindered the normal nucleation and the growth of the neighboring copper crystals. Consequently, the content of pure copper metal in the electrodeposited copper films decreased, and the intensities of diffraction peaks effectively reduced.

\subsection{Quantitative determination of glyphosate}

Based on the above evidences, we conducted a preliminary study for the quantitative determination of glyphosate. Different concentrations of glyphosate were added to the same concentration of $\mathrm{CuSO}_{4}$ electrolyte solution as additives in the same electrodeposition conditions. Due to the strong interaction between glyphosate and $\mathrm{Cu}^{2+}$ ions, the intensities of diffraction peaks of the deposited copper films on the ITO substrate surface decreased with the increase in the glyphosate concentration (Fig. 4). In this experiment, the quantitative detection of glyphosate was achieved by using the response of diffraction peak intensities and the glyphosate concentration based on the (111) lattice planes in the X-ray diffraction pattern of the deposited copper films mostly affected by glyphosate. Until now, there was no report on the quantitative detection of glyphosate by X-ray diffraction and electrochemical deposition. As can be seen from Fig. 9, the diffraction peak intensities of (111) lattice planes showed a good linear relationship with the logarithm value of glyphosate concentrations in the range from $1.0 \times 10^{-10} \mathrm{~mol} \mathrm{~L}^{-1}$ to $1.0 \times 10^{-5} \mathrm{~mol} \mathrm{~L}^{-1}\left(R^{2}=0.9958\right)$ with a detection limit of $2.5 \times 10^{-11} \mathrm{~mol} \mathrm{~L}^{-1}$. This method achieved a lower LOD value in comparison with those of the previous researches listed in Table $\mathrm{S} 1 . \dagger$ These results demonstrated that this method based on the combination of electrochemical deposition and X-ray diffraction provided a simple and effective method for the detection of glyphosate.

To demonstrate the selectivity of the method for glyphosate, we investigated the influence of possible interferences on the Xray diffraction peaks. The interferences, such as acetochlor (Ace), carbendazim (Car), $\mathrm{NO}_{3}{ }^{-}, \mathrm{SO}_{4}{ }^{-}$and $\mathrm{Cl}^{-}$, were detected (see Fig. S1 $\dagger$ ). The concentration of glyphosate was $1.0 \times$ $10^{-7} \mathrm{~mol} \mathrm{~L}^{-1}$ and that of others was $1.0 \times 10^{-5} \mathrm{~mol} \mathrm{~L}^{-1}$. The experimental results indicated that the above-mentioned interferences had no significant effects on the peak intensities of the X-ray diffraction patterns. The glyphosate molecule, containing $\mathrm{P}=\mathrm{O}, \mathrm{C}=\mathrm{O}$ and $\mathrm{N}-\mathrm{H}$ chelating groups, has a stronger affinity towards $\mathrm{Cu}^{2+}$ ions than other common pesticides. ${ }^{36}$

In order to prove the method feasibility in real sample analysis, recovery tests were employed by adding different amounts of glyphosate into milk samples. The results are shown in Table 3. The concentration of glyphosate was calculated according to the calibration curve. The average recovery range of glyphosate was from $95.0 \%$ to $103.4 \%(n=3)$. The results indicated that this method has potential application in the detection of glyphosate in milk samples.

\section{Conclusions}

In conclusion, the characterization of the electrodeposited copper film, added with glyphosate, showed that different concentrations of glyphosate had a significant effect on the morphology of the copper films. We conducted preliminary experiments on the application of this method for glyphosate detection. And using the target analyte as the additive makes the method expected for future applications. It can probe nonelectroactive substances that cannot be detected by classical electrochemical methods and broadens the scope of application of conventional X-ray diffraction measurements. The chelating reaction between the glyphosate and $\mathrm{Cu}^{2+}$ ions inhibited the reduction and growth of copper crystals. The microstructure and the morphology of the electrodeposited copper films were transformed, and the relative intensities of diffraction peaks decreased. This method can be used by any portable electrochemical workstation and X-ray diffractometer in the future, and can realize real-time field detection with good application prospects.

\section{Conflicts of interest}

There are no conflicts to declare.

\section{Acknowledgements}

This work was supported by the National Natural Science Foundation of China (Grant No. 21375116 and No. 16KJA150008), a project funded by the Priority Academic 
Program Development of Jiangsu Higher Education Institutions, and Jiangsu Province research program on analytical methods and techniques on the shared platform of large-scale instruments and equipment (BZ 201409). Results of STEM and XPS in the experiment were provided by Test Center of Yangzhou University.

\section{References}

1 T. Böcker, W. Britz and R. Finger, Ecol. Econ., 2018, 145, 182193.

2 C. M. Benbrook, Environ. Sci. Eur., 2016, 28, 3.

3 Y. D. Zebral, L. R. Lansini, P. G. Costa, M. Roza, A. Bianchini and R. B. Robaldo, Chemosphere, 2018, 196, 260-269.

4 A. H. C. Van Bruggen, M. M. He, K. Shin, V. Mai, K. C. Jeong, M. R. Finckh and J. G. Morris, Sci. Total Environ., 2018, 616617, 255-268.

5 S. H. Tseng, Y. W. Lo, P. C. Chang, S. S. Chou and H. M. Chang, J. Agric. Food Chem., 2004, 52, 4057-4063.

6 T. Arkan, A. Csámpai and I. Molnár-Perl, Microchem. J., 2016, 125, 219-223.

7 T. Arkan and I. Molnár-Perl, Microchem. J., 2015, 121, 99-106. 8 P. Szternfeld, S. V. Malysheva, V. Hanot and L. Joly, Food Anal. Method., 2016, 9, 1173-1179.

9 L. J. Marek and W. C. Koskinen, Pest Manage. Sci., 2014, 70, 1158-1164.

10 M. I. C. Marinho, M. F. Cabral and L. H. Mazo, J. Electroanal. Chem, 2012, 685, 8-14.

11 M. H. Do, A. Florea, C. Farre, A. Bonhomme, F. Bessueille, F. Vocanson, N.-T. Tran-Thi and N. Jaffrezic-Renault, Int. J. Environ. Anal. Chem., 2015, 95, 1489-1501.

12 B. B. Prasad, D. Jauhari and M. P. Tiwari, Biosens. Bioelectron., 2014, 59, 81-88.

13 A. Steinborn, L. Alder, B. Michalski, P. Zomer, P. Bendig, S. A. Martinez, H. G. Mol, T. J. Class and N. C. Pinheiro, J. Agric. Food Chem., 2016, 64, 1414-1421.

14 C. Rao and D. Trivedi, Coord. Chem. Rev., 2005, 249, 613-631.

15 A. Ramírez, P. Hillebrand, D. Stellmach, M. M. May, P. Bogdanoff and S. Fiechter, J. Phys. Chem. C, 2014, 118, 14073-14081.

16 L. Lee, P. Behera, K. R. Sriraman and R. R. Chromik, Wear, 2018, 400, 82-92.

17 A. Pei, G. Zheng, F. Shi, Y. Li and Y. Cui, Nano Lett., 2017, 17, 1132-1139.
18 G.-Q. Han, X. Shang, S.-S. Lu, B. Dong, X. Li, Y.-R. Liu, W.-H. Hu, J.-B. Zeng, Y.-M. Chai and C.-G. Liu, Int. J. Hydrogen Energy, 2017, 42, 5132-5138.

19 Z. Pu, Y. Luo, A. M. Asiri and X. Sun, ACS Appl. Mater. Interfaces, 2016, 8, 4718-4723.

20 S. Choe, B.-S. Lee, M. K. Cho, H.-J. Kim, D. Henkensmeier, S. J. Yoo, J. Y. Kim, S. Y. Lee, H. S. Park and J. H. Jang, Appl. Catal., B, 2018, 226, 289-294.

21 R. Akbari, G. Ramos Chagas, G. Godeau, M. Mohammadizadeh, F. Guittard and T. Darmanin, Appl. Surf. Sci., 2018, 443, 191-197.

22 J. C. Ballesteros, E. Chaînet, P. Ozil, G. Trejo and Y. Meas, J. Electroanal. Chem., 2010, 645, 94-102.

23 I. Gromyko, T. Dedova, S. Polivtseva, J. Kois, L. Puust, I. Sildos, A. Mere and M. Krunks, Thin Solid Films, 2018, 652, 10-15.

24 A. Bani Hashemi, G. Kasiri and F. La Mantia, Electrochim. Acta, 2017, 258, 703-708.

25 D. I. Tishkevich, S. S. Grabchikov, L. S. Tsybulskaya, V. S. Shendyukov, S. S. Perevoznikov, S. V. Trukhanov, E. L. Trukhanova, A. V. Trukhanov and D. A. Vinnik, J. Alloys Compd., 2018, 735, 1943-1948.

26 Y. J. Han, X. Zhang and G. W. Leach, Langmuir, 2014, 30, 3589-3598.

27 M. A. Pasquale, D. P. Barkey and A. J. Arvia, J. Electrochem. Soc., 2005, 105, 149-157.

28 S. Chhangani and M. J. N. V. Prasad, Mater. Charact., 2018, 136, 247-256.

29 B. Im and S. Kim, Microelectron. Eng., 2017, 172, 8-12.

30 C. Hesse, F. Goetz-Neunhoeffer and J. Neubauer, Cem. Concr. Res., 2011, 41, 123-128.

31 O. Lupan, T. Pauporté, L. Chow, B. Viana, F. Pellé, L. K. Ono, B. Roldan Cuenya and H. Heinrich, Appl. Surf. Sci., 2010, 256, 1895-1907.

32 R. Snellings, A. Bazzoni and K. Scrivener, Cem. Concr. Res., 2014, 59, 139-146.

33 L. Valentini, M. C. Dalconi, M. Favero, G. Artioli, G. Ferrari and G. Scherer, J. Am. Ceram. Soc., 2015, 98, 1259-1264.

34 M. Gui, J. Jiang, X. Wang, Y. Yan, S. Li, X. Xiao, T. Liu, T. Liu and Y. Feng, Sens. Actuators, B, 2017, 243, 696-703.

35 M. Rezaei, S. H. Tabaian and D. F. Haghshenas, Electrochim. Acta, 2012, 59, 360-366.

36 Y. Cao, L. N. Wang, C. Shen, C. Y. Wang, X. Y. Hu and G. X. Wang, Sens. Actuators, B, 2019, 283, 487-494. 